
\title{
25 Research Square \\ P2Y12 Inhibitor Utilization for Acute Coronary Syndrome in the Elderly: A Provincial Analysis From 2008 to 2018
}

\section{Saurabh Gupta}

McMaster University https://orcid.org/0000-0002-0114-0027

\section{Emilie P Belley-Cote}

McMaster University

\section{Adam Eqbal}

McMaster University

Charlotte McEwen

McMaster University

Ameen Basha

McMaster University

Nicole Wu

McMaster University

Joshua Cerasuolo

McMaster University

Shamir Mehta

McMaster University

Jon-David Schwalm

McMaster University

Richard P Whitlock ( $\nabla$ richard.whitlock@phri.ca )

McMaster University

Research

Keywords: ASA, ACS, CCS, CABG

Posted Date: October 29th, 2020

DOl: https://doi.org/10.21203/rs.3.rs-97757/v1

License: (c) (i) This work is licensed under a Creative Commons Attribution 4.0 International License.

Read Full License 
Version of Record: A version of this preprint was published at Implementation Science on April 21st, 2021. See the published version at https://doi.org/10.1186/s13012-021-01117-z. 


\section{Abstract}

Background Guidelines recommend acetylsalicylic acid (ASA) and ticagrelor following acute coronary syndrome (ACS), but appropriate prescription practices lag. We analyzed the impact of government medication approval, national guideline updates, and publicly funded drug coverage plans on P2Y12 inhibitor utilization.

Methods Accessing provincial databases, we obtained data for elderly ACS patients in Ontario, Canada between 2008 and 2018. Using an interrupted-time series, we evaluated types of P2Y12 inhibitors prescribed at discharge, and changes to their utilization following ticagrelor's national approval by Health Canada, national antiplatelet therapy guidelines (by the Canadian Cardiovascular Society (CCS)), and ticagrelor's coverage by a publicly funded medication plan.

Results We included 114,142 patients (49.4\%-PCl and 7.7\%-CABG). Proportion of PCl patients utilizing P2Y12 inhibitors increased from $73.4 \%$ to $86.9 \%(p<0.001)$ and $11.4 \%$ to $46.5 \%(p<0.001)$ for CABG patients. Among PCI patients, clopidogrel utilization declined monthly after 2010 national guidelines were published $(0.7 \% ; p<0.0001)$ and within the first month after ticagrelor's national approval by Health Canada (5.3\%; $p=0.03)$. Among PCI patients, ticagrelor utilization increased within the first month $(24.5 \%$; $p<0.0001)$ and continued increasing monthly $(0.4 \% ; p<0.0001)$ after its coverage by a publicly funded medication plan. Among CABG patients, ticagrelor's coverage was associated with a monthly increase in its utilization $(0.2 \% ; p<0.0001)$. Among PCl patients, clopidogrel utilization declined within the first month $(6.1 \% ; p=0.003)$ and ticagrelor utilization increased monthly $(0.3 \% ; p=0.05)$ after 2012 CCS guidelines.

Conclusion National guideline updates and drug coverage by a publicly funded medication plan significantly improved P2Y12 inhibitor utilization. Barriers to appropriate antiplatelet therapy in the surgical population must be explored.

\section{Contributions To The Literature}

- Significant gaps exist between evidence generation and implementation in cardiovascular health. Publishing new evidence rarely influences practitioners into adopting it.

- Using the knowledge-to-action framework, we discovered that policy changes - such as coverage by a publicly funded model - were essential in uptake of evidence.

- Personal beliefs around safety and efficacy, despite evidence and guidelines, continues to undermine implementation efforts. With help from facilitators, barriers to appropriate therapies must be addressed.

- Our findings contribute to already recognized gaps in the literature, including determining how and why evidence is used and who is most influenced.

\section{Introduction}


Based on large, multi-center randomized controlled trials (RCTs), guidelines recommend dual antiplatelet therapy (DAPT) with acetylsalicylic acid (ASA) and a P2Y12 inhibitor (specifically, ticagrelor) for 12 months after an acute coronary syndrome (ACS), regardless of management strategy: medical management, percutaneous intervention (PCI), or coronary artery bypass grafting (CABG).(1-10) (Fig. 1) Despite these recommendations and available evidence, appropriate DAPT use remains variable. For example, the ACS Reflective Program - a multicenter national observational registry conducted from 2011 to 2013 - found that clopidogrel remained the most common prescribed P2Y12 inhibitor across Canada at a rate of $82 \%$, compared to only $9 \%$ for ticagrelor. Further, the prospective Canadian Observational Antiplatelet Study (COAPT) demonstrated that despite RCTs and guideline recommendations, only a minority of ACS patients were discharged on ticagrelor $(11.1 \%)$ or prasugrel (5.7\%), compared to clopidogrel, after PCI. $(11,12)$ DAPT is particularly underused in patients undergoing CABG. In a cohort of patients with ACS, CABG was an independent predictor for DAPT underutilization (odds ratio [OR] 0.09, 95\% Cl 0.05-0.14).(13-15)

Suboptimal evidence implementation is not unique to antiplatelet therapy and ACS patients. Glynn et al. reviewed random patient samples from 12 metropolitans in the United States (U.S.) and found that less than $55 \%$ received guideline-recommended care.(16) More specific to cardiovascular health, a review of care gaps (defined as under-utilization or under-dosing of proven treatments) found that only $34-60 \%$ of eligible outpatients in Europe, Canada and U.S. received appropriate medications for heart failure, coronary artery disease or atrial fibrillation.(17) As such, implementation science strategies are paramount in improving healthcare delivery and quality of care.

To address the knowledge-implementation gap around antiplatelet therapy and ACS, we used provincial administrative data to examine P2Y12 inhibitor utilization after discharge of elderly ACS patients. We aimed to evaluate whether the preponderance of clopidogrel prescriptions among patients managed medically or with PCI still stands, and if CABG patients saw an increase in DAPT utilization. We also sought to determine how prescription practices varied between patients managed with PCl, CABG or medically, and how - if at all - knowledge publication, government approval, guideline recommendations and publicly funded medication plan coverage impacted practice patterns.

\section{Methods}

\section{Study Population}

We included all patients 65 years of age or older who presented with an ACS and were subsequently referred for coronary angiogram in the province of Ontario, Canada from October 1, 2008 (chosen as such due to availability of complete Cardiac Care Network (CCN) datasets from then onwards) to March 31, 2018. Ontario includes 20 cardiac centers, which provide care to 14.5 million inhabitants. Apart from universal healthcare, the provincial government provides a publicly-funded medication plan for those 65 years or older (Ontario Drugs Benefits (ODB)).

\section{Databases and Cohort Definition}


We obtained data on baseline characteristics and prescriptions filled from five databases linked at the Institute for Clinical Evaluation Sciences (ICES) - CorHealth, Ontario Drug Benefit (ODB) database, Discharge Abstract Database (DAD) and Same-Day Surgery Database (SDS) from Canadian Institute for Health Information (CIHI), National Ambulatory Care Reporting System (NACRS), and Registered Persons Database (RPDB). These databases, linked via unique encoded identifiers analyzed at ICES, were used to analyse health outcomes as individuals can be tracked over time.(18-21) Available data on admission for ACS, patient characteristics, and management strategies were captured retrospectively from the CCN (CorHealth) database.

Using variables from CCN, we applied the following cohort exclusions: aged younger than 65 years or older than 105 years (which was derived from RPDB), did not have a coronary angiogram within 3 months of ACS, primary reason for cardiac surgery referral was not coronary disease, died prior to discharge from facility performing coronary angiogram, normal anatomy on angiogram, or nonobstructive coronary artery disease. Definitions for all other clinical variables are available on the CCN website (https://www.corhealthontario.ca/data-\&-reporting/data-collection-\&-access/CCN-Registries-DataEntry-Reference-Manual-\&-Data-Standards-Document-Updated-September-2017.pdf). We identified patients undergoing PCI or CABG from DAD and SDS databases by ICD-10 codes: 1.IJ.50, 1.IJ.54 or 1.IJ.57 for $\mathrm{PCl}$ and 1.IJ.76 for CABG.

\section{Outcomes}

We tracked the number of P2Y12 inhibitor prescriptions filled within 14 days (per Ontario Primary Care Medication Reconciliation Guide, medications should be reconciled within 14 days from hospital discharge), based on treatment strategy. Eligible agents included ticagrelor, clopidogrel and prasugrel. We assumed that patients taking either one of these antiplatelet agents were also taking ASA, since it is available over the counter and cannot be tracked by ODB.

Our outcome of interest was change in prescription utilization at specific time points, decided by discussion among the authors and feedback from reviewers; approval of ticagrelor by Health Canada in June 2011, changes to the "Use of Antiplatelet Therapy in the Outpatient Setting: Canadian Cardiovascular Society (CCS) Guidelines" (herein called CCS Antiplatelet Therapy Guidelines) in May 2011 and November 2013, publication of "Prasugrel versus Clopidogrel for Acute Coronary Syndromes without Revascularization" (TRILOCY ACS) in October 2012, and publicly funded medication plan (ODB) coverage of ticagrelor for ACS patients in April 2013.

\section{Statistical Analysis}

We created an interrupted time series with the dataset aggregated on a monthly basis. The dependent variable was the proportion of patients dispensed a P2Y12 inhibitor within 14 days of hospital discharge. Our autoregressive model included the following covariates:

1. Intervention (medical, $\mathrm{PCl}$ or $\mathrm{CABG}$ ) - binary indicator denoting 0 during pre-intervention period and 1 during post-intervention period; 
2. time (in months) - ordinal indicator denoting months since start of study;

3. time since intervention (in months) - ordinal indicator denoting months since time interruption (i.e. implementation of intervention);

4. autoregressive processes (if applicable).

We also incorporated lag periods in our interrupted time series; they included the month of intervention and three months after (to allow for uptake of intervention into clinical practice). All data points enclosed in the lag period were excluded from analysis.

To build the model, we initially added 13 autoregressive parameters because the datasets were aggregated by month -13 processes allow the models to detect and adjust for seasonality. In a backward stepwise fashion, autoregressive parameters were removed until all autoregressive parameters remained statistically significant. Presence of autoregression (or autocorrelation) was assessed using Durbin-Watson statistic.(22) Presence of seasonality (stationarity) was assessed using Dickey-Fuller statistic.(23)

The output and data analysis for this study was generated using SAS software.

\section{Ethics approval}

The study was approved by the Hamilton Integrated Research Ethics Board (HiREB \#4196). ICES is an independent, non-profit research institute whose legal status under Ontario's health information privacy law allows it to collect and analyze health care and demographic data, without consent, for health system evaluation and improvement.

\section{Results}

\section{Population Characteristics}

Figure 1 represents the study flow diagram. We identified a total of 114,142 elderly patients and present their baseline characteristics in Table 1. PCl was performed in $49.4 \%$ of patients (mean age, $75.71 \pm 6.94$, $62.3 \%$ male), CABG in $7.7 \%$ of patients (mean age, $74.11 \pm 5.63 ; 73.5 \%$ male), and $42.9 \%$ were managed medically (mean age, $75.71 \pm 6.50 ; 62.9 \%$ male). Medically managed patients were more likely to have undergone previous interventions ( $\mathrm{PCl}$ and/or $\mathrm{CABG}$ ) and had more comorbidities with a higher proportion presenting with congestive heart failure (CHF), chronic obstructive pulmonary obstruction (COPD), cerebrovascular disease (CVD), and peripheral vascular disease (PVD). Further, medically managed patients were more likely to already be on antithrombotic medications before their ACS. At the beginning of our accrual period, the proportion of ACS patients prescribed P2Y12 inhibitors at discharge was $73.4 \%$ in the $\mathrm{PCl}$ group, $11.4 \%$ in the CABG group, and $31.4 \%$ in the medically managed group. As of March 2018 , the proportion had increased to $86.9 \%(p<0.001)$ for patients undergoing $\mathrm{PCl}, 46.5 \%(\mathrm{p}<$ $0.001)$ for those undergoing $C A B G$, and $32.3 \%(p=0.775)$ for those managed medically. 
Table 1

- Characteristics of ACS Patients Stratified by Management Strategy

\begin{tabular}{|lllll|}
\hline Characteristic & Medical Management & PCI & CABG & p-value \\
& $\mathbf{n = 4 9 , 6 5 2}$ & $\mathbf{n = 5 6 , 3 5 8}$ & $\mathbf{n = 8 , 1 3 2}$ & \\
\hline Age & $75.71 \pm 6.50$ & $75.71 \pm 6.94$ & $74.11 \pm 5.63$ & $<.001$ \\
\hline Male Sex (\%Male) & $30,742(61.9 \%)$ & $35,137(62.3 \%)$ & $5,973(73.5 \%)$ & $<.001$ \\
\hline Current Smoker & $6,521(13.1 \%)$ & $8,010(14.2 \%)$ & $1,272(15.6 \%)$ & NS \\
\hline Previous Smoker & $17,301(34.8 \%)$ & $16,532(29.3 \%)$ & $2,866(35.2 \%)$ & $<.001$ \\
\hline COPD & $5,276(10.6 \%)$ & $4,552(8.1 \%)$ & $598(7.4 \%)$ & $<.001$ \\
\hline CVD & $5,723(11.5 \%)$ & $5,240(9.3 \%)$ & $809(9.9 \%)$ & $<.001$ \\
\hline CHF & $7,157(14.4 \%)$ & $4,962(8.8 \%)$ & $812(10.0 \%)$ & $<.001$ \\
\hline Diabetes & $18,193(36.6 \%)$ & $16,737(29.7 \%)$ & $2,855(35.1 \%)$ & $<.001$ \\
\hline Hyperlipidemia & $37,018(74.6 \%)$ & $34,964(62.0 \%)$ & $5,418(66.6 \%)$ & $<.001$ \\
\hline Hypertension & $39,566(79.7 \%)$ & $40,309(71.5 \%)$ & $6,025(74.1 \%)$ & $<.001$ \\
\hline History of MI & $18,988(38.2 \%)$ & $15,950(28.3 \%)$ & $1,947(23.9 \%)$ & $<.001$ \\
\hline PVD & $5,583(11.2 \%)$ & $4,533(8.0 \%)$ & $839(10.3 \%)$ & $<.001$ \\
\hline Renal Disease & $261(0.5 \%)$ & $215(0.4 \%)$ & $31(0.4 \%)$ & NS \\
\hline Previous CABG & $10,643(21.4 \%)$ & $8,042(14.3 \%)$ & $251(3.1 \%)$ & $<.001$ \\
\hline Previous PCl & $11,722(23.6 \%)$ & $12,284(21.8 \%)$ & $977(12.0 \%)$ & $<.001$ \\
\hline Previous Antithrombotic: & & & & $<.001$ \\
\hline Dabigatran & $744(1.5 \%)$ & $630(1.1 \%)$ & $58(0.1 \%)$ & $<.001$ \\
\hline Apixaban & $779(1.6 \%)$ & $795(1.4 \%)$ & $66(0.8 \%)$ & NS \\
\hline Rivaroxaban & $992(2.0 \%)$ & $1,156(2.1 \%)$ & $116(1.4 \%)$ & $<.001$ \\
\hline Warfarin & $4,894(9.9 \%)$ & $3,700(6.6 \%)$ & $396(4.9 \%)$ & 0.01 \\
\hline Prasugrel & $21(0.0 \%)$ & $36(0.1 \%)$ & $(0.0 \%)$ & \\
\hline
\end{tabular}

*Mean \pm SD.

Abbreviations: COPD - Chronic Obstructive Pulmonary Disease; CVD - Cerebrovascular Disease; CHF - Congestive Heart Failure; MI - myocardial infarction; PVD - Peripheral Vascular Disease; CABG Coronary Artery Bypass Grafting Surgery; PCl - Percutaneous Coronary Intervention.

The baseline characteristics, including medical co-morbidities and previous antithrombotic use, were measured when patient presented with their ACS. 


\begin{tabular}{|lllll|}
\hline Characteristic & $\begin{array}{l}\text { Medical Management } \\
\mathbf{n = 4 9 , 6 5 2}\end{array}$ & $\begin{array}{l}\text { PCI } \\
\mathbf{n = 5 6 , 3 5 8}\end{array}$ & $\begin{array}{l}\text { CABG } \\
\mathbf{n = 8 , 1 3 2}\end{array}$ & P-value \\
\hline Clopidogrel & $11,575(23.3 \%)$ & $10,614(18.8 \%)$ & $1,029(12.7 \%)$ & $<.001$ \\
\hline Ticagrelor & $589(1.2 \%)$ & $992(1.8 \%)$ & $45(0.6 \%)$ & $<.001$ \\
\hline *Mean \pm SD. & & & \\
\hline $\begin{array}{l}\text { Abbreviations: COPD - Chronic Obstructive Pulmonary Disease; CVD - Cerebrovascular Disease; CHF } \\
\text { - Congestive Heart Failure; Ml - myocardial infarction; PVD - Peripheral Vascular Disease; CABG - } \\
\text { Coronary Artery Bypass Grafting Surgery; PCl - Percutaneous Coronary Intervention. }\end{array}$ & \\
\hline $\begin{array}{l}\text { The baseline characteristics, including medical co-morbidities and previous antithrombotic use, were } \\
\text { measured when patient presented with their ACS. }\end{array}$ & \\
\hline
\end{tabular}

While we evaluated prasugrel utilization independently, the number of patients discharged on prasugrel was extremely low, and we were unable to conduct a robust analysis; the models did not converge and could not be interpreted.

Figure 2 - our central image - reviews all key findings for PCI and CABG patients as detailed below.

\section{P2Y12 Inhibitor Utilization and 2010 CCS Antiplatelet Therapy Guidelines (Fig. 3)}

We could not evaluate the impact of the 2010 CCS Antiplatelet Therapy Guidelines on ticagrelor because it was not yet approved by ODB.

\section{PCI Patients}

The publication of the 2010 CCS Antiplatelet Therapy Guidelines in May 2011 was associated with a significant decline of $6.1 \%(p=0.003)$ in utilization of all available P2Y12 inhibitors within the first month. The trend continued, but not significantly, in the months after. Individual clopidogrel utilization also declined within the first month after guideline updates, albeit not significantly. Clopidogrel utilization, however, declined significantly by $0.7 \%(p<0.0001)$ every month thereafter.

\section{CABG Patients}

The publication of 2010 CCS Antiplatelet Therapy Guidelines in May 2011 was associated with a decline in utilization of all available P2Y12 inhibitors within the first month and every month thereafter, however this was not significant. Clopidogrel utilization demonstrated similar trends, which were also not significant.

\section{Medically Managed Patients}


The publication of 2010 CCS Antiplatelet Therapy Guidelines was associated with a significant decline of $3.8 \%(p=0.002)$ in utilization of all available P2Y12 inhibitors within the first month, followed by a significant decline of $0.2 \%(p=0.002)$ every month after. Individually, the guideline updates also impacted clopidogrel utilization within the first month and were associated with a significant decline of $2.5 \%(p=$ $0.02)$, followed by a significant decline of $0.3 \%(p<0.0001)$ every month after.

\section{P2Y12 Inhibitor Utilization and Ticagrelor Health Canada Approval (Fig. 3)}

We could not evaluate the impact of ticagrelor's Health Canada approval on ticagrelor prescriptions itself as it was not approved by ODB yet.

\section{PCl Patients}

The approval of ticagrelor by Health Canada in June 2011 was associated with a significant decline of $7.3 \%(p<0.002)$ in utilization of all available P2Y12 inhibitors within the first month, and while the trend continued monthly thereafter, it was not significant. Utilization of clopidogrel declined by $5.3 \%(p=0.03)$ within the first month, and continued to decline every month thereafter, but not significantly.

\section{CABG Patients}

The approval of ticagrelor by Health Canada in June 2011 demonstrated a decline in utilization of all available P2Y12 inhibitors, but this was not significant. The trend continued every month thereafter, without reaching statistical significance. Clopidogrel utilization showed similar trends, which also failed to reach statistical significance.

\section{Medically Managed Patients}

The approval of ticagrelor by Health Canada in June 2011 was associated with a significant decline of $4.3 \%(p<0.001)$ in utilization of all available P2Y12 inhibitors within the first month and continued to significantly decline monthly thereafter by $0.2 \%(p<0.001)$. Clopidogrel utilization also declined by $3.2 \%$ $(p=0.003)$ within the first month and continued to significantly decline every month thereafter by $0.3 \%$ ( $p$ $<0.001)$.

\section{P2Y12 Inhibitor Utilization and Coverage of Ticagrelor by the Publicly Funded Medication Plan (Fig. 3)}

\section{PCI Patients}

Ticagrelor's coverage by the publicly funded medication plan in August 2013 was associated with an increase of $11.4 \%(p<0.0001)$ in utilization of all available P2Y12 inhibitors within the first month after coverage began, and while the trend continued every month after, it was not significant. The approval was associated with a significant decline of $14 \%(p<0.0001)$ in clopidogrel utilization, followed by a decline 
of $0.2 \%(p=0.03)$ every month thereafter. Meanwhile, ticagrelor utilization increased by $24.5 \%(p<$ $0.0001)$ within the first month and continued to increase by $0.4 \%$ every month $(p<0.0001)$ thereafter.

\section{CABG Patients}

Ticagrelor's coverage by the publicly funded medication plan in August 2013 was not associated with a significant change in utilization of all available P2Y12 inhibitors, or clopidogrel and ticagrelor individually, within the first month. However, after, there was a significant increase of $0.3 \%(p=0.0001)$ every month in utilization of all available P2Y12 inhibitors. With the coverage, clopidogrel utilization also increased every month after by $0.2 \%(p=0.04)$. Meanwhile, ticagrelor utilization also increased by $0.2 \%(p<0.0001)$ every month after its approval.

\section{Medically Managed Patients}

Ticagrelor's approval by the publicly funded medication plan in August 2013 was not associated with a significant impact on utilization of all available P2Y12 inhibitors. Individually, the approval was associated with a significant decline of $0.1 \%(p=0.005)$ in clopidogrel utilization every month after. Meanwhile, ticagrelor utilization demonstrated a significant increase of $2.2 \%(p<0.0001)$ within the first month after its approval and continued to significantly increase by $0.12 \%(p<0.0001)$ every month after.

\section{P2Y12 Inhibitor Utilization and 2012 CCS Antiplatelet Therapy Guidelines (Fig. 3)}

\section{PCI Patients}

The publication of 2012 CCS Antiplatelet Therapy Guidelines in August 2013 was associated with a significant increase of 7.4\% ( $p=0.0004)$ in utilization of all available P2Y12 inhibitors within the first month after publication. The trend continued in the subsequent months but was not significant. The updated guidelines were associated with a decline of $6.1 \%(p=0.003)$ in clopidogrel utilization within the first month after publication, followed by a significant decline of $0.4 \%(p=0.002)$ every month thereafter. Meanwhile, ticagrelor utilization demonstrated an increase within the first month, albeit not significant. Thereafter, ticagrelor utilization significantly increased by $0.3 \%(p=0.05)$ every month.

\section{CABG Patients}

With the publication of 2012 CCS Antiplatelet Therapy Guidelines in August 2013, utilization of all available P2Y12 inhibitors decreased by 5.2\% ( $p=0.05)$ within the first month but did not demonstrate a significant trend every month thereafter. While clopidogrel utilization also declined within the first month, and monthly afterwards, the change was not significant. Similarly, while ticagrelor utilization increased every month after guideline publication, the change was not significant.

\section{Medically Managed Patients}

The publication of 2012 CCS Antiplatelet Therapy Guidelines was not associated with a significant change in utilization of all available P2Y12 inhibitors within the first month or after. Individually, the 
guideline updates were also not associated with a significant change in clopidogrel utilization. After the 2012 guideline updates, ticagrelor utilization, however, demonstrated a significant increase of $1.7 \%(p<$ 0.0001) within the first month. This trend continued every month after but was not significant.

\section{Discussion}

We analysed P2Y12 inhibitor utilization data on 114,142 elderly ACS patients, and identified three key evidence-practice gaps. First, the uptake of evidence is slow and although the proportion of patients receiving P2Y12 inhibitors after an ACS has improved in the last decade, the proportion of patients receiving guideline-directed therapy with ticagrelor remains low. Second, DAPT use is suboptimal for patients who undergo CABG. Third, and perhaps most importantly, policies have the biggest influence on changing practice. Drug coverage by a publicly funded medication plan and guideline updates had the largest impact on appropriate medication utilization.

DAPT has proven benefit for ACS patients undergoing medical management, $\mathrm{PCl}$ or CABG.(6,7) An RCT of 18,624 patients randomized to ASA and ticagrelor demonstrated that the latter reduced the primary composite outcome of vascular mortality, myocardial ischemia (MI) or cerebrovascular accidents (CVA) $9.8 \%$ with ASA and ticagrelor vs $11.75 \%$ with ASA and clopidogrel (HR $0.84 ; 95 \% \mathrm{Cl} 0.77-0.92)$ at $12-$ months.(6) Previously, Yusuf et al. compared DAPT with ASA and clopidogrel with ASA monotherapy and demonstrated that a composite of death from cardiovascular causes, non-fatal MI or stroke occurred in 9.3\% of patients receiving DAPT and in $11.4 \%$ of patients receiving ASA only (RR $0.80 ; 95 \% \mathrm{Cl} 0.72-0.90$; $p<0.001)$.(7) The results of CABG subgroups from both above trials were also consistent with above findings, demonstrating a reduction in major adverse cardiovascular events (MACE) when using more potent antiplatelet therapy regimens.(24,25) Recently, Zhao et al. randomized 500 CABG patients to 1:1:1 (ticagrelor and ASA: ticagrelor monotherapy: ASA monotherapy) and reported a statistically significant increase in vein graft patency when comparing DAPT with ticagrelor and ASA to ASA monotherapy alone (12.2\% [95\% Cl, 5.2-19.2\%]; $\mathrm{p}$ < 0.001).(9) In a recent network meta-analysis, Gupta et al. studied the safety and efficacy of various antiplatelet regimens in 15,511 CABG patients and reported that DAPT with ASA and ticagrelor, compared to ASA monotherapy, reduced SVG stenosis (OR 0.40; 95\% Credible Interval (Crl) $0.21,0.74)$, mortality (OR 0.52; 95\% Crl 0.30, 0.87) and MACE (OR 0.63; 95\% Crl 0.44, 0.91).(26,27) As such, DAPT is an evidence-based and guideline-recommended treatment for patients with ACS regardless of the management approach.

Despite this, P2Y12 inhibitor prescription practices lag globally; our data corroborate this. We demonstrated that at end of our accrual window in March 2018, only $86.9 \%$ of PCl patients were discharged with P2Y12 inhibitors. This is not dissimilar to the previously published real-world data; Schwalm et al. reported a 92\% compliance in P2Y12 inhibitor prescriptions after PCl in a cluster RCT, while and Turgeon et al. demonstrated a $80.5 \%$ compliance rate in their cohort of 13,897 ACS patients treated with $\mathrm{PCl}$ in Alberta from 2012 to 2016.(28,29) Esposti et al. analysed discharge prescriptions of 1,882 ACS patients in Italy. Of the $83 \%$ of patients who were discharged on any antiplatelet therapy, $57 \%$ were prescribed ASA and clopidogrel and only $0.1 \%$ of patients were discharged on ASA and ticagrelor. 
(30) DAPT is even further underutilized in ACS patients who undergo CABG compared to PCl. In a retrospective study of 8,939 ACS patients in Australia, CABG was an independent predictor for DAPT underutilization; OR $0.09,95 \% \mathrm{Cl} 0.05-0.14$.(13) A recent survey evaluating the practice patterns of 75 Canadian cardiac surgeons around postoperative antiplatelet management found that only $45 \%$ of cardiac surgeons would restart DAPT in patients who suffered a recent ACS. Interestingly, they were more likely to initiate DAPT in patients who had previous stents to vessels that were not bypassed, required endarterectomy, or suffered a peri-operative MI. Respondents (81\%) were more concerned with preventing bleeding than recurrent ischemic events.(31)

While underutilization of ticagrelor post-ACS in our cohort may appear concerning, we must note that our population is elderly, with a mean age over 74, and use of clopidogrel may be justified. Recently, the POPular AGE Trial (Clopidogrel versus ticagrelor or prasugrel in patients aged 70 years or older with nonST-elevation acute coronary syndrome) demonstrated that ticagrelor was prematurely discontinued in $47 \%$ of patients (due to bleeding and dyspnea) compared to clopidogrel, which was only discontinued in $22 \%$ of patients. The authors also concluded that clopidogrel was an appropriate alternative to ticagrelor among ACS patients aged 70 years or older.(32) Within Canada, the Alberta Provincial Project for Outcome Assessment in Coronary Heart Disease registry evaluated clopidogrel versus ticagrelor prescriptions and outcomes in ACS patients who were discharged after a PCI. In their cohort of 11,185 PCI patients, compared with clopidogrel, ticagrelor was not associated with lower risk of MACE (aHR 0.97; 95\% Cl 0.85-1.10), but was associated with an increased risk of major bleeding (aHR 1.51; 95\% Cl 1.291.78) and dyspnea (aHR 1.98; 95\% Cl 1.47-2.65).(29) These findings introduce a nuance to bleeding and dyspneic risk with ticagrelor, which seems particularly pronounced among the elderly, potentially explaining why clopidogrel remains so widely used in our cohort.

Additionally, it should be noted that evidence for use of DAPT after CABG has significant limitations RCTs are small or are sub-studies of larger RCTs. Furthermore, there is significant population heterogeneity within the RCTs on whether patients underwent off-pump CABG (OPCAB) or CABG with cardiopulmonary bypass. In fact, a large majority of patients randomized in the two trials demonstrating DAPT superiority over ASA monotherapy underwent OPCAB. $(33,34)$ The available evidence does not evaluate the use of DAPT in patients undergoing multiple arterial grafting, which are associated with significantly better patency rates and clinical outcomes compared with vein grafts. and the effect DAPT has on the latter may not apply to arterial grafts. $(35,36)$ Given the limitation of existing evidence, physicians and surgeons are more concerned with post-procedural bleeding risk, lack of dedicated robust evidence in CABG patients.

Given the above findings and real-world data, the discordance between guidelines and practice is likely multifactorial. The underutilization of ticagrelor may be due to some of the barriers to physician adherence discussed by Cabana et al. Specifically, with ever-expanding research, physicians may find it challenging to keep abreast with updated guidelines or apply them due to previous practice inertia. While lack of familiarity with ticagrelor (pharmacology and evidence around it) or external barriers (perceived issues with costs and drug coverage) may play a role, the most likely factors are likely due to lack of 
outcome expectancy (belief in efficacy) and lack of agreement (belief in safety, especially fear of increased bleeding, and belief in strength of recommendations).(37) In fact, the fear of post-procedural bleeding may also explain why all P2Y12 inhibitor utilization among CABG patients declined significantly after the 2012 CCS Antiplatelet Therapy Guidelines were published. Despite strong recommendations for ASA and ticagrelor, surgeons were likely not convinced of the efficacy and safety in this population.(11)

Our observation that provincial coverage increases prescription practice is aligned with the results of other studies and highlights the importance of health benefits and provincial formulary coverage. In 2011, Quebec became the first province in Canada to cover ticagrelor and prasugrel after an ACS; on discharge, Quebec showed increased use of ticagrelor and prasugrel (37.1\%) compared to rest of Canada (19.2\%). (11) In 2008, Jackevicius et al. published on the impact of ODB coverage and clopidogrel use among patients who underwent $\mathrm{PCl}$ after an acute $\mathrm{MI}$ in Ontario; demonstrating an increase from $35 \%$ utilization before coverage to $88 \%$ after coverage in the first 30 days after discharge $(p<0.001)$. (38) Though adherence to guideline-directed use of DAPT following ACS will never reach $100 \%$, targeted knowledge translation strategies are required to improve antiplatelet management for ACS patients in Ontario, and around the world. As per the practical knowledge translation framework, a national survey to understand barriers towards appropriate antiplatelet prescription is required.(39) First, this would help address the underutilization of ticagrelor as compared to clopidogrel, and would aim to address the subset of ACS patients undergoing CABG, where surgeons are least adherent to prescribing guideline-directed DAPT. Increased prescription compliance in this population could close the practice gap and improve secondary prevention for these patients. Lastly, knowledge translation strategies should be developed during the medication study period (the pre-trial results stage) to expedite uptake if the study is positive.

\section{Limitations}

Our study has several limitations. First, we included patients who had a coronary angiogram within three months after an ACS. Given this, there is a potential for survival bias in our cohort assembly since some patients may have died before undergoing an angiogram and may represent a population with different prescription patterns. While we discuss P2Y12 inhibitor prescription practices in our study, it should be noted that patient compliance is a significant limitation of our study. That is, our data capture is dependent on patients filling their prescriptions, and not whether physicians prescribed the appropriate medication. Similarly, not all patients are compliant with over the counter ASA, and the inability to capture how many patients were taking this also remains a limitation. Last, $24.5 \%$ of medically managed patients, $20.7 \%$ of $\mathrm{PCl}$ patients, and $13.3 \%$ of CABG patients were on $\mathrm{P} 2 \mathrm{Y} 12$ inhibitors before presenting with an ACS. Presumably, if these patients were continued on the same, they may not have required a prescription refill within the 14-days after discharge, leading to a miscount of actual P2Y12 inhibitor utilization. Lastly, a significant number of patients were on more potent anticoagulants (either a vitamin $\mathrm{K}$ antagonist or a direct oral anticoagulant), and may present a higher risk for bleeding, contraindicating the addition of a P2Y12 inhibitor, especially given the elderly age of our cohort. 
Furthermore, the study design was such that it did not capture patients under the age of 65 , nor capture prescriptions not covered by ODB. Due to this, we did not capture the prescription utilization of younger ACS patients. And we can only make assumptions about ticagrelor utilization rates prior to ODB coverage based on the decline demonstrated in utilization of other P2Y12 inhibitors. For instance, the drop in clopidogrel and all available P2Y12 inhibitor prescriptions within the month after ticagrelor's Health Canada approval and the 2010 CCS Antiplatelet Therapy Guideline publication likely reflects an increase in out-of-pocket (paid-for by patients) ticagrelor prescriptions. Furthermore, the use of "Brilinta Savings Card", which allowed some patients to pay as low as $\$ 5$ for a 30 day supply of ticagrelor after Health Canada approval, along with samples from drug companies, most likely led to the decline in utilization of all available P2Y12 inhibitors, as more patients switched to ticagrelor. However, we cannot confirm this hypothesis.

\section{Conclusion}

Our study shows an overall increase in utilization of all available P2Y12 inhibitors for elderly ACS patients compared to previous Canadian national registries. Despite guideline recommendations and definitive evidence, gaps exist in management of ACS patients for which we must identify barriers to inform future knowledge translation interventions. Policy changes appear to be most effective at moving clinicians towards guideline-directed medical therapy.

\section{Declarations}

\section{Ethics Approval and Consent to Participate}

The study was approved by the Hamilton Integrated Research Ethics Board (HiREB \#4196).

No individual patient consent to participate was required.

\section{Consent for Publication}

Not applicable.

\section{Availability of Data and Materials}

The data that support the findings of this study are available from ICES, but restrictions apply to the availability of these data, which were used under licensure for the current study, and so are not publicly available. Data are however available from the authors upon reasonable request and with permission of ICES.

Note: ICES is an independent, non-profit research institute whose legal status under Ontario's health information privacy law allows it to collect and analyze health care and demographic data, without consent, for health system evaluation and improvement.

\section{Competing Interests}


Dr Shamir Mehta is a consultant for AstraZeneca and receives grant support from them.

All the other authors do not have any competing interests; financial or otherwise.

\section{Funding}

This study was conducted with grant support awarded to Dr Saurabh Gupta by Hamilton Health Sciences - NIF-17425. Additionally, this study was supported by ICES, which is funded by an annual grant from the Ontario Ministry of Health and Long-Term Care (MOHLTC).

\section{Author Contributions}

SG made substantial contributions to the conception and design of the work; acquisition and interpretation of data; drafted the work and substantially revised it.

EBC made substantial contributions to the conception and design of the work; and substantially revised it.

AE made substantial contributions to interpretation of data; drafted the work and substantially revised it. CE made substantial contributions to interpretation of data; drafted the work and substantially revised it. $A B$ and NW made substantial contributions to drafted the work and substantially revised it. JC made substantial contributions to data acquisition, analysis and interpretation of data. SM, JDS made substantial contributions to design of the work and substantially revised it. RW made substantial contributions to contributions to the conception and design of the work; substantially revised it; and supervised all efforts.

All authors approve the submitted version.

All authors agree to be personally accountable for the author's own contributions and to ensure that questions related to the accuracy or integrity of any part of the work, even ones in which the author was not personally involved, are appropriately investigated, resolved, and the resolution documented in the literature.

\section{Acknowledgements}

The authors would like to thank Richard Perez of ICES McMaster for helping coordinate the data capture and analysis. Parts of this material are based on data and information compiled and provided by $\mathrm{ClHI}$. The analyses, conclusions, opinions, and statements expressed herein are solely those of the authors and do not reflect those of the funding or data sources; no endorsement is intended or should be inferred. The authors acknowledge that the clinical registry data used in this publication is from participating hospitals through CorHealth Ontario, which serves as an advisory body to the Ministry of Health and Long-Term Care (MOHLTC), is funded by the MOHLTC, and is dedicated to improving the quality, efficiency, access, and equity in the delivery of the continuum of adult cardiac, vascular, and stroke services in Ontario, Canada. We thank IMS Brogan Inc. for use of their Drug Information Database. 


\section{References}

1. Amsterdam EA, Wenger NK, Brindis RG et al. 2014 AHA/ACC Guideline for the Management of Patients with Non-ST-Elevation Acute Coronary Syndromes: a report of the American College of Cardiology/American Heart Association Task Force on Practice Guidelines. J Am Coll Cardiol 2014;64:e139-e228.

2. Hamm CW, Bassand JP, Agewall S et al. ESC Guidelines for the management of acute coronary syndromes in patients presenting without persistent ST-segment elevation: The Task Force for the management of acute coronary syndromes (ACS) in patients presenting without persistent STsegment elevation of the European Society of Cardiology (ESC). Eur Heart J 2011;32:2999-3054.

3. Mehta SR, Bainey KR, Cantor WJ et al. 2018 Canadian Cardiovascular Society/Canadian Association of Interventional Cardiology Focused Update of the Guidelines for the Use of Antiplatelet Therapy. Can J Cardiol 2018;34:214-233.

4. Arnett DK, Blumenthal RS, Albert MA et al. 2019 ACC/AHA Guideline on the Primary Prevention of Cardiovascular Disease: A Report of the American College of Cardiology/American Heart Association Task Force on Clinical Practice Guidelines. J Am Coll Cardiol 2019.

5. Valgimigli M, Bueno H, Byrne RA et al. 2017 ESC focused update on dual antiplatelet therapy in coronary artery disease developed in collaboration with EACTS: The Task Force for dual antiplatelet therapy in coronary artery disease of the European Society of Cardiology (ESC) and of the European Association for Cardio-Thoracic Surgery (EACTS). Eur Heart J 2018;39:213-260.

6. Wallentin L, Becker RC, Budaj A et al. Ticagrelor versus clopidogrel in patients with acute coronary syndromes. N Engl J Med 2009;361:1045-57.

7. Yusuf S, Zhao F, Mehta SR et al. Effects of clopidogrel in addition to aspirin in patients with acute coronary syndromes without ST-segment elevation. N Engl J Med 2001;345:494-502.

8. Wiviott SD, Antman EM, Gibson CM et al. Evaluation of prasugrel compared with clopidogrel in patients with acute coronary syndromes: design and rationale for the TRial to assess Improvement in Therapeutic Outcomes by optimizing platelet InhibitioN with prasugrel Thrombolysis In Myocardial Infarction 38 (TRITON-TIMI 38). Am Heart J 2006;152:627-35.

9. Zhao Q, Zhu Y, Xu Z et al. Effect of Ticagrelor Plus Aspirin, Ticagrelor Alone, or Aspirin Alone on Saphenous Vein Graft Patency 1 Year After Coronary Artery Bypass Grafting: A Randomized Clinical Trial. JAMA 2018;319:1677-1686.

10. Tanguay JF, Bell AD, Ackman ML et al. Focused 2012 update of the Canadian Cardiovascular Society guidelines for the use of antiplatelet therapy. Can J Cardiol 2013;29:1334-45.

11. Gandhi S, Zile B, Tan MK et al. Increased uptake of guideline-recommended oral antiplatelet therapy: insights from the Canadian acute coronary syndrome reflective. Can J Cardiol 2014;30:1725-31.

12. Dery JP, Mehta SR, Fisher HN et al. Baseline characteristics, adenosine diphosphate receptor inhibitor treatment patterns, and in-hospital outcomes of myocardial infarction patients undergoing 
percutaneous coronary intervention in the prospective Canadian Observational AntiPlatelet sTudy (COAPT). Am Heart J 2016;181:26-34.

13. Anastasius M, Lau JK, Hyun K et al. The underutilisation of dual antiplatelet therapy in acute coronary syndrome. Int J Cardiol 2017;240:30-36.

14. Benedetto U, Altman DG, Gerry S et al. Impact of dual antiplatelet therapy after coronary artery bypass surgery on 1-year outcomes in the Arterial Revascularization Trial. Eur $\mathrm{J}$ Cardiothorac Surg 2017;52:456-461.

15. Bomb R, Oliphant CS, Khouzam RN. Dual Antiplatelet Therapy After Coronary Artery Bypass Grafting in the Setting of Acute Coronary Syndrome. Am J Cardiol 2015;116:148-54.

16. McGlynn EA, Asch SM, Adams $\mathrm{J}$ et al. The quality of health care delivered to adults in the United States. N Engl J Med 2003;348:2635-45.

17. Majumdar SR, McAlister FA, Furberg CD. From knowledge to practice in chronic cardiovascular disease: a long and winding road. J Am Coll Cardiol 2004;43:1738-42.

18. Guru V, Fremes SE, Austin PC, Blackstone EH, Tu JV. Gender differences in outcomes after hospital discharge from coronary artery bypass grafting. Circulation 2006;113:507-16.

19. Hassan A, Newman A, Ko DT et al. Increasing rates of angioplasty versus bypass surgery in Canada, 1994-2005. Am Heart J 2010;160:958-65.

20. Silaruks S, Thinkhamrop B, Tantikosum W, Wongvipaporn C, Tatsanavivat P, Klungboonkrong V. A prognostic model for predicting the disappearance of left atrial thrombi among candidates for percutaneous transvenous mitral commissurotomy. J Am Coll Cardiol 2002;39:886-91.

21. Whitlock R, Healey JS, Connolly SJ et al. Predictors of early and late stroke following cardiac surgery. CMAJ 2014;186:905-11.

22. Smith DP. Durbin-Watson statistics for model life tables. Asian Pac Cens Forum 1983;9:7-9.

23. Moineddin R, Upshur RE, Crighton E, Mamdani M. Autoregression as a means of assessing the strength of seasonality in a time series. Popul Health Metr 2003;1:10.

24. Fox KA, Mehta SR, Peters $R$ et al. Benefits and risks of the combination of clopidogrel and aspirin in patients undergoing surgical revascularization for non-ST-elevation acute coronary syndrome: the Clopidogrel in Unstable angina to prevent Recurrent ischemic Events (CURE) Trial. Circulation 2004; 110:1202-8.

25. Held C, Asenblad N, Bassand JP et al. Ticagrelor versus clopidogrel in patients with acute coronary syndromes undergoing coronary artery bypass surgery: results from the PLATO (Platelet Inhibition and Patient Outcomes) trial. J Am Coll Cardiol 2011;57:672-84.

26. Gupta S, Belley-Cote EP, Rochwerg B et al. Antiplatelet therapy and coronary artery bypass grafting: Protocol for a systematic review and network meta-analysis. Medicine (Baltimore) 2019;98:e16880.

27. Gupta SB-C, E. Panchal, P. Pandey, A. Rochwerg, B. Mehta, S. Schwalm, J. Whitlock, R.P. Antiplatelet therapy and coronary artery bypass grafting: a systematic review and network meta-analysis. Interact Cardiov Th 2020. 
28. Schwalm JD, Ivers NM, Natarajan MK et al. Cluster randomized controlled trial of Delayed Educational Reminders for Long-term Medication Adherence in ST-Elevation Myocardial Infarction (DERLA-STEMI). Am Heart J 2015;170:903-13.

29. Turgeon RD, Koshman SL, Youngson E et al. Association of Ticagrelor vs Clopidogrel With Major Adverse Coronary Events in Patients With Acute Coronary Syndrome Undergoing Percutaneous Coronary Intervention. JAMA Intern Med 2020.

30. Degli Esposti L, Perrone V, Veronesi C, Buda S, Rossini R, a LG. Long-Term Use of Antiplatelet Therapy in Real-World Patients with Acute Myocardial Infarction: Insights from the PIPER Study. TH Open 2018;2:e437-e444.

31. Yanagawa B, Ruel M, Bonneau $\mathrm{C}$ et al. Dual antiplatelet therapy use by Canadian cardiac surgeons. J Thorac Cardiovasc Surg 2015;150:1548-54 e3.

32. Gimbel M, Qaderdan K, Willemsen $\mathrm{L}$ et al. Clopidogrel versus ticagrelor or prasugrel in patients aged 70 years or older with non-ST-elevation acute coronary syndrome (POPular AGE): the randomised, open-label, non-inferiority trial. Lancet 2020;395:1374-1381.

33. Mannacio VA, Di Tommaso L, Antignan A, De Amicis V, Vosa C. Aspirin plus clopidogrel for optimal platelet inhibition following off-pump coronary artery bypass surgery: results from the CRYSSA (prevention of Coronary arteRY bypaSS occlusion After off-pump procedures) randomised study. Heart 2012;98:1710-1715.

34. Zhao Q, Zhu YP, Xu ZY et al. Effect of Ticagrelor Plus Aspirin, Ticagrelor Alone, or Aspirin Alone on Saphenous Vein Graft Patency 1 Year After Coronary Artery Bypass Grafting A Randomized Clinical Trial. Jama-Journal of the American Medical Association 2018;319:1677-1686.

35. Leonard JR, Di Franco A, Gaudino M. Dual antiplatelet therapy post CABG?-perhaps, but... why not a radial artery instead? J Thorac Dis 2018;10:S2106-S2108.

36. Gupta SB-C, E. Agahi, P. Basha, A. Jaffer, I. Mehta, S. Schwalm, J. Whitlock, R.P. . Antiplatelet Therapy and Coronary Artery Bypass Grafting: Analysis of current evidence with a focus on acute coronary syndrome. Can J Cardiol 2019.

37. Cabana MD, Rand CS, Powe NR et al. Why don't physicians follow clinical practice guidelines? A framework for improvement. JAMA 1999;282:1458-65.

38. Jackevicius CA, Tu JV, Demers $V$ et al. Cardiovascular outcomes after a change in prescription policy for clopidogrel. N Engl J Med 2008;359:1802-10.

39. Licskai C, Sands T, Ong M, Paolatto L, Nicoletti I. Using a knowledge translation framework to implement asthma clinical practice guidelines in primary care. Int J Qual Health Care 2012;24:53846.

\section{Figures}




\begin{tabular}{|c|c|c|c|}
\hline \multicolumn{3}{|c|}{914964} & Ontario residents in CCN with valid IKN \\
\hline \multicolumn{4}{|c|}{$\downarrow$} \\
\hline \multicolumn{3}{|c|}{462240} & Patients aged $65-105$ \\
\hline \multicolumn{4}{|c|}{$\downarrow$} \\
\hline \multicolumn{3}{|c|}{292080} & Patients who underwent coronary angiogram \\
\hline \multicolumn{4}{|c|}{$\downarrow$} \\
\hline \multicolumn{3}{|c|}{266635} & Primary reason for referral: $C A D$ \\
\hline \multicolumn{4}{|c|}{$\downarrow$} \\
\hline \multicolumn{3}{|c|}{137094} & Patients presented with ACS \\
\hline \multicolumn{3}{|c|}{134090} & Patients alive at discharge \\
\hline \multicolumn{4}{|c|}{$\downarrow$} \\
\hline \multicolumn{3}{|c|}{116728} & Obstructive CAD \\
\hline \multicolumn{4}{|c|}{$\downarrow$} \\
\hline \multicolumn{3}{|c|}{114142} & Overlapping CCN records available for analysis \\
\hline \multicolumn{4}{|c|}{$\downarrow$} \\
\hline Medical Mgmt & $\mathrm{PCl}$ & CABG & \multirow{2}{*}{ Cohorts created using $\mathrm{CCl}$ from $\mathrm{ClHI}-\mathrm{DAD} / \mathrm{SDS}$} \\
\hline 49652 & 56358 & 8132 & \\
\hline
\end{tabular}

Figure 1

Study flow diagram demonstrating development of cohorts. 


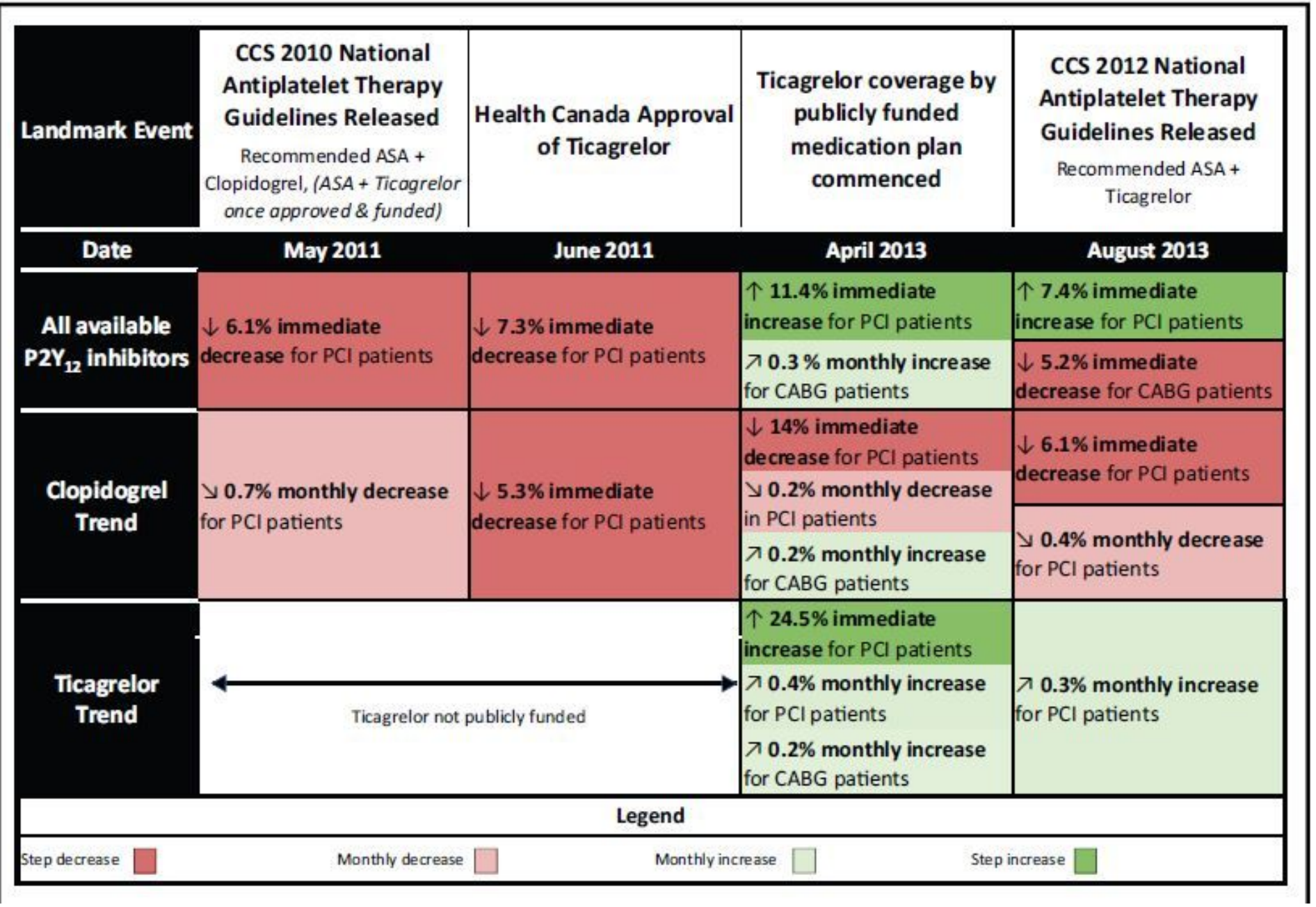

Figure 2

Drug coverage by a publicly funded medication plan and guideline updates had significant impact on P2Y12 inhibitor prescription practices. 

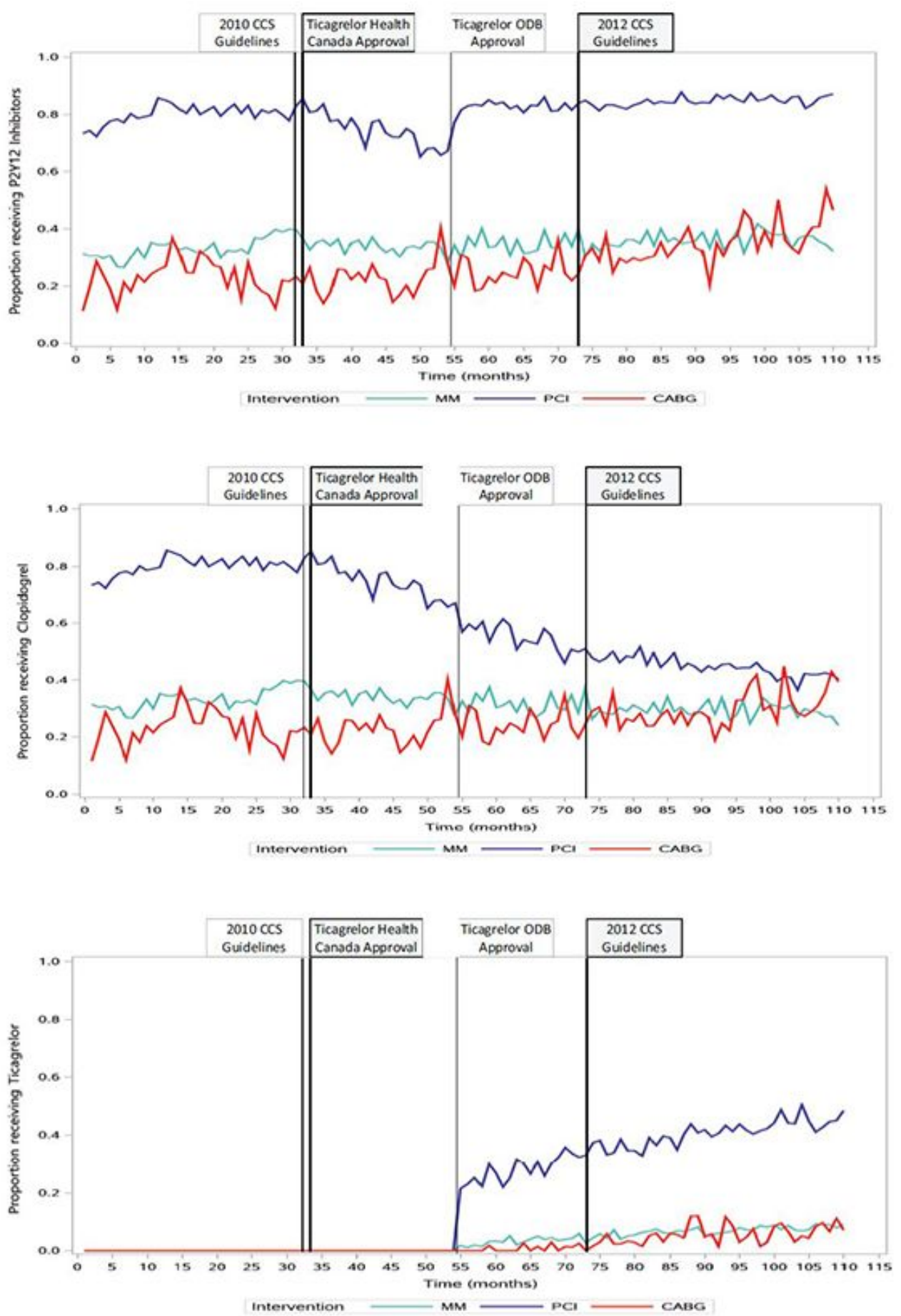

\section{Figure 3}

a. Changes in utilization of all available P2Y12 inhibitors from 2008 to 2018 . Each time point of interest was used as an intercept to analyse for significant changes in practice patterns. MM - Medically managed; $\mathrm{PCl}$ - percutaneous intervention; $\mathrm{CABG}$ - coronary artery bypass grafting. $b$. Changes in Clopidogrel utilization from 2008 to 2018. Each time point of interest was used as an intercept to analyse for significant changes in practice patterns. MM - Medically managed; $\mathrm{PCl}$ - percutaneous intervention; 
CABG - coronary artery bypass grafting. c. Changes in Ticagrelor utilization from 2008 to 2018. Each time point of interest was used as an intercept to analyse for significant changes in practice patterns. $\mathrm{MM}$ - Medically managed; $\mathrm{PCl}$ - percutaneous intervention; $\mathrm{CABG}$ - coronary artery bypass grafting.

\section{Supplementary Files}

This is a list of supplementary files associated with this preprint. Click to download.

- STROBEchecklistv4combined.docx 